\title{
Non-interactive Localization of Cognitive Radios Based on Dynamic Signal Strength Mapping
}

\author{
Song Liu*, Yingying Chen ${ }^{\dagger}$, Wade Trappe* and Larry J. Greenstein* \\ ${ }^{*}$ WINLAB, Rutgers University \\ North Brunswick, NJ 08902 \\ Email: \{song, trappe, ljg\}@winlab.rutgers.edu \\ ${ }^{\dagger}$ Dept. of ECE, Stevens Institute of Technology \\ Hoboken, NJ 07030 \\ Email: yingying.chen@stevens.edu
}

\begin{abstract}
The openness of the lower-layer protocol stacks in cognitive radios increases the flexibility of dynamic spectrum access and promotes spectrally-efficient communications. To ensure the effectiveness of spectrum sharing, it is desirable to locate primary users, secondary users, and unauthorized users in a non-interactive fashion based on limited measurement data at receivers. In this work, we present two range-free localization algorithms based on dynamic mapping of received signal strength (RSS) to perform non-interactive localization that does not require the cooperation from the cognitive device to be located. A fine-grained signal strength map across the surveillance area is constructed dynamically through interpolation. By making use of this signal map, the proposed schemes can achieve higher accuracy of location estimation than existing non-interactive and RSS based methods in most channel variation conditions. Both our simulation results as well as testbed evaluations have demonstrated the feasibility of the proposed algorithms.
\end{abstract}

\section{INTRODUCTION}

The openness of the lower-layer protocol stacks renders cognitive radios an appealing solution to dynamic spectrum access. Its open nature will increase the flexibility of spectrum utilization and promote spectrally-efficient communications. In the new paradigm of opportunistic spectrum access driven by cognitive radios, the location awareness has its special importance to issues such as spectrum etiquette enforcement [1] and spectrum hole discovery [2]. The knowledge of locations of incumbent transmitters including both primary users and secondary users makes it possible to predict the potential spectrum hole without on-site measurements, and thus more agile spectrum sharing strategies can be performed. On the other hand, to guarantee the effectiveness of a cooperative spectrum sharing, it is necessary that the spectrum management unit has the ability to locate unauthorized transmitters.

Although a great effort has been devoted to localization techniques in a variety of radio systems [3]-[6], the aforementioned localization tasks limit the application of most existing algorithms in the new paradigm of spectrum access. Because we do not expect incumbent users or unauthorized users to provide any cooperation during localization. Thus, the position estimation in cognitive radio systems has to be conducted in a non-interactive fashion, based on limited measurement data at receivers. Further, due to the nature of the wireless transmission in cognitive radios, the most accessible information at a receiver is the received signal strength (RSS). This motivates us to investigate non-interactive localization methods employing RSS in a cognitive radio framework.
Given the radio propagation law in nature, the RSS usually corresponds to the direct link distance between a transmitter and a receiver, with random distortions by obstacles, multipath and noises. Such a relationship can be exploited to directly deduce the link distance [3], or can be translated to some coarser but less sensitive metrics, such as connectivity or area to bound candidate positions [4], [5]. The former is known as range-based localization and the latter range-free. To achieve non-interactive localization for networks with cognitive radios, range-free approaches are more feasible. Further, we may improve the location accuracy using range-free based on the following observations:

- Most of studies [7] have shown that a higher location accuracy can be achieved by a finer-grained measurement;

- The RSS among neighboring locations always exhibit some level of correlations, which can be characterized by certain propagation models [8].

By making use of such correlations, it is possible to recover more RSS readings without actual measurement. In this work, we explore the potential benefit of measurement data correlations to localization employing RSS. We propose two interpolation-based algorithms, namely Interpolation-based Weighted Centroid (IWC) and Interpolation-based Point-inTriangle (IPIT). As their names imply, the proposed algorithms utilize a set of fine-grained RSS data generated by interpolation in realtime, which we call a dynamic signal strength map. The use of interpolation in localization is not new and it has been shown to improve the accuracy in fingerprint matching algorithms [3]. However, there is little work available that provides a way to apply interpolation on instantaneously measured signal strength so as to improve the accuracy of noninteractive localization. Our proposed algorithms demonstrate the potential advantage of this dynamic fine-grained RSS mapping. Moreover, without involving an off-line training phase compared to fingerprint matching methods, our algorithms are less time- and labor-consuming and more robust against a volatile radio environment. We note that our algorithms can be extended beyond cognitive radios and easily applied to the localization of any wireless transmitting devices provided to have RSS measurements available.

To evaluate the localization performance of our approaches in both outdoor and indoor environments, we performed simulations of an outdoor environment and conducted exper- 
iments in a testbed within an indoor building environment. We compared the performance of our methods to existing localization approaches under different channel conditions and network topologies. Our results demonstrated that our algorithms can extract extra information from limited RSS readings by interpolation and thus achieve higher location accuracy than existing methods.

The rest of the paper is organized as follows: Section II reviews existing RSS based localization algorithms. Section III describes the propagation model which is the theoretical basis of the paper. Section IV and Section V present our proposed algorithms, IWC and IPIT, respectively. We evaluate the performance of the algorithms compared to existing algorithms by simulation and testbed experiments in Section VI and VII, respectively. Finally, Section VIII concludes our work.

\section{RELATED WORK}

Among a variety of localization techniques, RSS based approaches have attracted many interests as a cost-saving solution, since RSS information is accessible in most offthe-shelf radio devices and allows the reuse of existing infrastructures with minimum extra cost [6]. With regard to the techniques of location estimation employing RSS, the existing algorithms are generally categorized as ranged-based and range-free solutions.

Conventional range-based methods for non-interactive localization estimate the absolute link distance between the unknown location and the given location of a receiver, which is usually called an anchor ${ }^{1}$. Provided multiple distance estimates collected at a central processor, the unknown location can be pinpointed by variant algorithms such as the lateration, minmax, or Euclidean [9], [10]. These methods are also integrated into collaborative strategies to infer multi-hop distances in distributed localizations for large-scale sensor networks [11] [13]. Direct distance estimation requires a preassumption of the radio propagation model, which is hard to obtain under hostile channel conditions. Thus, the localization estimations are sensitive and less reliable.

On the other hand, range-free approaches [4], [14]-[16] have less stringent hardware requirement and less dependence on the exact propagation properties. They have been pursued as cost-effective, coarse-grained but reliable solutions.

The centroid estimation is a typical scheme for range-free localization. It represents a class of methods, all of which use the center of a polygon as the estimate location. [4] proposed an out-door localization system based on the proximity information. In this system, anchors with established positions transmitted periodic beacon signals and nodes located themselves using the average of the locations of proximate anchors. This simple form of centroid is robust against environmental variations but resulting in a crude approximation. Provided a low resolution of anchors or a mild channel condition, a weighted version of the centroid method usually has a higher accuracy [10].

${ }^{1}$ From now on, we use an anchor to denote a locator with known location, and a node denotes the device to be located, in accord with existing studies.
Further, APIT (Approximate Point-in-Triangle), proposed in [14], is an area-based localization scheme for large-scale sensor networks with high node density. It selects groups of three anchors that have radio connectivity to a sensor node with unknown location and forms a triangle for each group. For each triangle, the node decides if it is within the triangle based on signal strength measured by itself and neighboring nodes. The location of the node can be pinpointed to the intersection area of all the triangles that contain the node. Since APIT requires exchanges of RSS measurements among multiple nodes within a fairly small range, its performance is sensitive to the node density. In addition, the node itself is responsible for estimating the location and thus the approach does not apply to the aforementioned non-interactive localization tasks. [15] proposed a similar area-based approach using ring-overlapping, which does not require collaborations among nodes. However, it also requires the node to perform the localization function.

In addition to the two localization categories discussed above, there is a type of location estimation more suitable to indoor environments, namely, RSS fingerprint matching [3], [7]. In these methods, a calibration phase (off-line) is conducted to build a RSS fingerprint map for a set of known locations. Then during the on-line phase, an unknown location is determined by searching for the closest match between the run-time RSS measurement and the fingerprints in the map. However, the off-line calibration is a both time-consuming and labor-intensive process, and these algorithms are often less scalable.

\section{Propagation MOdel}

We assume the RSS of an anchor (i.e., a cognitive radio or a sensor) at location $\mathrm{x}$ from a transmitting node at location $\mathbf{x}_{0}$, is given by the following propagation model [17]:

$$
P(\mathbf{x})=P_{0}-10 \gamma \log _{10}\left(\frac{\left\|\mathbf{x}-\mathbf{x}_{0}\right\|}{d_{0}}\right)+s(\mathbf{x}) \quad(\mathrm{dB}),
$$

where $P_{0}$ is the received signal strength, in $\mathrm{dB}$, at the reference distance $d_{0} . \gamma$ is the path loss exponent. $s(\mathbf{x})$ accounts for the shadow fading between the transmitter and the anchor, and is widely modeled as a Gaussian random process over space with zero mean and standard deviation $\sigma_{d B}$. Although many research efforts on localization were based on the preassumption that the shadowing is an i.i.d. process among different locations, experimental results have provided evidence that shadowing is a spatially correlated process [8], [18]. [8] also proposed a handy exponential model describing the autocorrelation of this spatial process,

$$
E\left[s\left(\mathbf{x}_{i}\right) s\left(\mathbf{x}_{j}\right)\right]=\sigma_{d B}^{2} e^{-\left\|\mathbf{x}_{i}-\mathbf{x}_{j}\right\| / X_{C}},
$$

where the correlation distance $X_{C}$ ranges from several to many tens of meters, and the standard deviation $\sigma_{d B}$ from $3 \mathrm{~dB}$ to $10 \mathrm{~dB}$ in outdoor environments [8].

As depicted in the above model, we do not specify the direction of a propagation link. The assumption of our proposed algorithms is that, the antenna at each device (i.e., a transmitter 
or a receiver) has a constant gain across all directions. That is, the antenna gain is assumed omnidirectional.

Although we use a particular path loss model here for the purpose of evaluating our approaches, our methods do not rely on the validity of this or any other models. Our methods utilize interpolation to create "virtual anchors" that are combined with actual anchors to provide inputs for location estimation. The proposed algorithms are variants of two localization schemes, namely, the weighted centroid and APIT, as described in Section II. Both of our algorithms are range-free (do not rely on the knowledge of the propagation law) and non-interactive (require no cooperation from the transmitter to be localized). We present our algorithms in the next two sections.

\section{INTERPolation-BASED Weighted CENTROID (IWC)}

In this section, we present the Interpolation-based Weighted Centroid algorithm for localization.

\section{A. Weighted Centroid}

Centroid localization, proposed in [4], estimates the location of a node by the centroid of all anchors' positions within its communication range. Without considering the distance between the node and anchors, the method is robust against environmental uncertainties but often results in a coarse estimation. An enhanced version, which weighs the distance from anchors, has been shown to be more accurate in practice [10].

In the weighted centroid approach, the RSS, known to be a function of link distance, is used as a weighing factor to average the locations of neighboring anchors, the location of a node can be approximated by

$$
\mathbf{X}=\sum_{i=1}^{N} \frac{P_{i}}{\sum_{j=1}^{N} P_{j}} \mathbf{X}_{i},
$$

where $P_{i}$ is the linear received power at the $i$ th-anchor with the coordinate $\mathbf{X}_{i}$. It has been observed that the weighted centroid method has a monotonically increasing accuracy as the resolution of anchors increases [10]. Though a practical system usually has the RSS measurements only in a limited number of positions, we may obtain more estimated values by making use of the interpolation techniques and the intrinsic correlation among the RSS data as a result of the propagation properties. It is thus interesting to investigate how these estimated RSS measurements can affect the localization accuracy.

\section{B. Interpolation}

In our scheme, we use radial base functions (RBF) [19] for interpolation. We note that our approach can be easily extended to use any 2-D interpolation techniques. The RBF method constructs the approximation of a surface by solving the linear equations:

$$
s(\mathbf{x})=p(\mathbf{x})+\sum_{i=1}^{N} \lambda_{i} \phi\left(\left\|\mathbf{x}-\mathbf{x}_{i}\right\|\right), \quad \mathbf{x} \in \mathbb{R}^{2},
$$

where $p(\mathbf{x})$ is a polynomial of degree at most $k, \phi$ is a continuous basis function, $\phi: \mathbb{R}^{+} \rightarrow \mathbb{R}$, and $N$ is the number

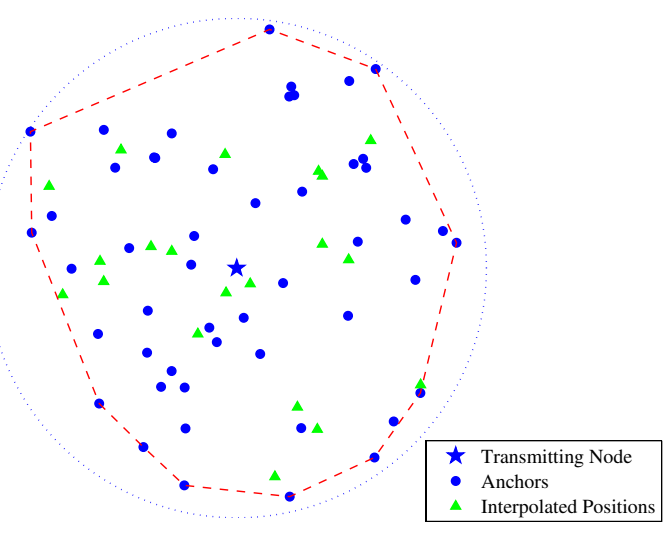

Fig. 1. Interpolation-based Weighted Centroid (IWC): A number of positions are randomly selected within the convex hull bounded by the neighboring anchors of the transmitter. The RSS values at these positions are estimated by interpolation.

of given data (i.e., actual RSS measurements at $N$ anchors). By solving the coefficient $\lambda_{i}$ and the polynomial $p(\mathbf{x})$, the value at any point can be approximated by an explicit function. To minimize the computational cost, we use the biharmonic spline, $\phi(r)=r$, and the linear polynomial $p(\mathbf{x})$ (i.e., $k=1$ ) in our algorithm. Since the surface defined by the signal strength in logarithmic scale does not vary as much as the one in the linear scale, we will use the former to construct the interpolant.

\section{Localization}

Suppose there are $N$ anchors uniformly distributed within the communication range of a transmitting node. Before applying location estimation as described in (3), we randomly select $N_{\text {intp }}$ positions within the coverage area of these $N$ anchors, i.e., the convex area delimited by the anchors as shown in Fig. 1. The RSS values at these $N_{\text {intp }}$ positions are approximated via interpolation. Both the interpolated and measured data are then applied to the weighted centroid algorithm (3). In reality, the anchors may not be uniformly distributed around the node in a network, for example, when the node is close to the edge of the coverage area. Previous work has shown that an unbalanced distribution of anchors can degrade the accuracy of a centroid based algorithm [4]. To mitigate the impact due to the unbalance of network topology, we choose $N_{n b}<N$ anchors with the strongest RSS readings to apply the localization algorithm. Based on the propagation property, these $N_{n b}$ anchors should be the closest neighbors of the transmitting node, and thus the location accuracy will be less affected by the geographic limitation of the network.

\section{Interpolation-BASED POINT-IN-TRIANGle (IPIT)}

In this section, we present the Interpolation-based Point-inTriangle (IPIT) algorithm for localization. Similar to APIT [14], IPIT is an intersection-area based method and uses triangles to narrow down the location of the node. However, an interpolation approach is utilized to assist the point-intriangle decision and IPIT does not require cooperation of 


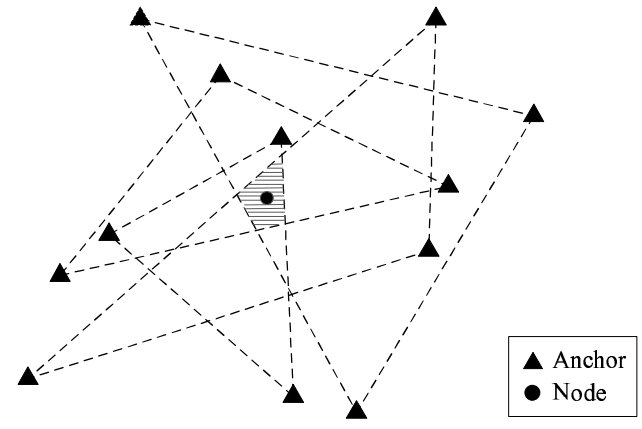

Fig. 2. APIT (Approximate Point-In-Triangle) algorithm locates a node within the intersection of overlapping triangles. The black triangles denote anchors and the black dot denotes the node to be located.

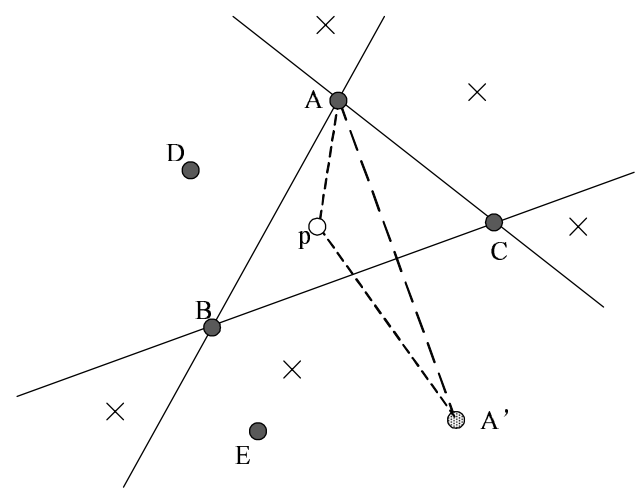

Fig. 3. IPIT (Interpolation based PIT) algorithm. The point-in-triangle test is performed by comparing the measured RSS at every anchor (e.g., A) and the estimated RSS at its mirror point (e.g., $A^{\prime}$ ) with respect to the opposite edge (e.g., $B C$ ).

nodes to perform location estimation so that the new algorithm is suitable to the non-interactive localization tasks.

\section{A. PIT test by symmetric vertexes}

PIT (Point-in-Triangle) test is the basic component of both IPIT and APIT algorithms. As explained in its name, PIT test identifies anchor triplets that encompass the node. Then the location of the node can be pinpointed to the intersection area of all these triangles. As illustrated in Fig. 2, in the existing APIT algorithm, a candidate anchor triplet is determined based on signal strength measured by the node and its neighboring nodes. Consequently, the node itself is responsible for performing the test. Next, we show that, by using a geometry property, PIT test can be done at the anchors without cooperation from the node. Thus, the proposed algorithm IPIT can be applied to non-interactive localization. We illustrate our approach by examining Co-side Condition and PIT Condition.

Co-side Condition: As illustrated in Fig. 3, a point $p$ and the vertex $A$ of a triangle $\triangle A B C$ are on the same side of the edge $B C$ if $p$ is closer to $A$ than to $A$ 's symmetric point with respect to the edge $B C$ (i.e., $A^{\prime}$ ).

Note that the co-side condition applies to all three vertices of a triangle. For convenience, we call a point and a vertex are "co-side" if the above condition holds. Now, we have a necessary and sufficient condition that a node $p$ is inside a triangle $\triangle A B C$ as:

PIT Condition: if $p$ and every vertex of a triangle $\triangle A B C$ are co-side, $p$ is inside $\triangle A B C$.

The distance from a node $p$ to any point is related to the path loss of the link between them, as defined in (1). Since a point closer to the node is more likely to have a higher power reading, the closer link can be determined by choosing the one with the higher received power. Although an anchor at a vertex (e.g., $A$ ) can obtain the signal-strength information by direct measurement, we do not expect to have another anchor being placed exactly at the symmetric point (e.g., $A^{\prime}$ ). Thus, we use the same interpolation technique as in the IWC algorithm to obtain an estimated RSS at this symmetric point.

\section{B. Voting for PIT Test}

The key step in the PIT test is the co-side test. That is, we need to determine for every vertex (i.e. anchor) of a triangle whether it is on the same side with the node to be located. Due to deep fading or inaccurate interpolation, an anchor closer to the node does not necessarily have a large RSS and then a wrong conclusion may be drawn. To mitigate the estimation error in IPIT caused by hostile channel conditions and interpolation inaccuracy, we propose a voting scheme for the decision of PIT.

Suppose there are $N$ anchors and a node $p$ whose location is unknown. Now we want to find out whether an anchor $A$ and $p$ are co-side with respect to an edge $B C$, as depict in Fig.3. Apparently we will apply the co-side condition on the anchor $A$. Nevertheless, we can also apply the co-side condition on any of the $N-3$ anchors other than $A, B$ and $C$. For example, we use the co-side condition on an anchor $D$ and find out it is on the same side with $p$. Since all anchors' locations are given, we know exactly whether the anchor $A$ and $D$ are on the same side with respect to the edge $B C$. Thus, by estimating the coside relationship of $D$ and $p$, we can also tell whether $A$ and $p$ are co-side. Specifically, with respect to $B C$, the following conditions hold:

1) If $A$ and $D$ are co-side:

\{ If $D$ and $p$ are co-side, $A$ and $p$ are co-side;

\{ If $D$ and $p$ are not co-side, $A$ and $p$ are not co-side.

2) If $A$ and $D$ are not co-side:

\{ If $D$ and $p$ are co-side, $A$ and $p$ are not co-side;

\{ If $D$ and $p$ are not co-side, $A$ and $p$ are co-side.

Therefore, by applying the co-side condition on the $N-2$ anchors other than $B$ and $C$, we have $N-2$ answers for the co-side relationship of $A$ and $p$. Then, we choose the answer of the majorities as the final decision ${ }^{2}$.

\section{Localization}

The IPIT test is performed for every combination of three anchors and then each triangle has an indicator to mark whether the node is inside or outside. The node most likely

\footnotetext{
${ }^{2}$ In the current algorithm, we discard a triangle if we do not have a majority answer for a co-side test.
} 
falls within the intersection by the most triangles. Since it is computational prohibitive to find the analytical geometry of the intersection, a grid-scan algorithm [5] is used to approximate the area. In this algorithm, the entire area is divided into small grids. Each grid has an initial value 0. If the node is within a triangle, all grids inside the triangle increase their values by 1 ; otherwise, decrease by 1 . Then, the intersection area is approximated by the set of grids with the maximum score. The location of the node is estimated by the centroid of the area.

The complete IPIT algorithm is summarized as follows:

1) After the anchors are deployed, their location information is collected by a central processor or a powerful computing node that runs the localization algorithm. For any line defined by two anchors, the processor computes the symmetric points for all anchors and store them in a table. Suppose there are total $N$ anchors, the size of the table is $(N-2) C_{N}^{2}$, which is about $O\left(N^{3}\right)$;

2) All anchors measure the RSS from the node with unknown location and then report the RSS value (in $\mathrm{dB}$ ) to the central processor. All the following steps are done by the processor only;

3) The RSS at all symmetric points are estimated by interpolation;

4) Find all combinations of three anchor locations, which yields $C_{N}^{3}$ triangles;

5) For each edge of a triangle, perform the voting process to determine whether the opposite vertex is on the same side of the unknown node with respect to the edge. If two sides have the same votes, a decision is not made and the triangle is discarded. If the node is co-side with vertexes for all three edges, the PIT condition is satisfied and thus the node is in the triangle;

6) After polling all the triangles, calculate the intersection area by the most number of triangles using the gridscan algorithm and estimate the node's location by the centroid of the area.

The basic algorithm is computationally expensive. In practice, however, we can reduce the complexity in several steps. The symmetric point calculation in Step 1 is required only once throughout the duty as long as no new anchor joins. Further, the symmetric points can be discarded if they are outside the convex hull that encloses all the anchors, since values at those points are approximated by extrapolation, which usually has high errors. This can greatly reduce the number of co-side tests. Additionally, we can limit the number of triangles polled in Step 5. In the following simulations, the test will stop if we find 200 triangles that enclose the node.

\section{Simulation Results}

In this section, we first describe our experimental setup to simulate an outdoor environment. We then present simulation results that evaluate the effects of different parameters in IWC on localization performance. Next, we compare the localization results of our proposed algorithms, IWC and IPIT, with a set of representative localization methods.

\section{A. Simulation settings}

We assume anchors are randomly deployed in the surveillance area. An unknown transmitting node has a transmission range $R$, so that all anchors within $R$ can detect the signal from the node. Specifically, anchors are uniformly distributed within the circular area centered by the node. In the current study, we do not consider the effect of the network edge as mentioned in Section IV-C. That is, we assume the size of the network is sufficiently large and the location of the node can be optimistically approximated to the center of its coverage area. We denote the number of anchors in the circle by $N$. Note that the transmission range $R$ is only used for normalization. We assume an out-door environment and a path loss exponential $\gamma$ of 3.8 in our simulations. The performance of the localization algorithms is evaluated in terms of the localization estimation error, which is defined as the Euclidian distance between the true location and the estimated one. The simulation is run for 5000 times and the anchor locations and the channel variation (i.e., the shadowing) are randomly generated in each trial. The root mean square error (RMSE) of the estimation is computed over all trials and normalized by $R$.

\section{B. Parameter Study in IWC}

The IWC method has two adjustable parameters, the number of neighboring anchors $N_{n b}$ and the number of interpolated positions $N_{\text {intp }}$. In the original weighted centroid method, it has been shown that the location accuracy is insensitive to $N_{n b}$ [10]. Our simulation results show this is also true for IWC with a sufficiently large $N$. We plotted the estimation RMSE under different values of $N_{n b}$ in Fig. 4 for Weighted Centroid and IWC, respectively. The total number of anchors $N$ ranges from 10 to 100 . Here, $\sigma_{d B}=0,4$ and $8 \mathrm{~dB}$ represent perfect, moderate and high variation of the shadowing fading, respectively. In this test, we chose $N_{\text {intp }}=500$ when $N_{n b}=N$ and fixed the density of interpolating points in the area. That is, for any $N_{n b}<N$, the actual number of interpolating points also proportionally decreases (i.e., $\sim N_{\text {intp }} \cdot N_{n b} / N$ ). We observed that the localization error of IWC monotonically decreases with the increasing of $N_{n b}$ and the performance stabilizes when $N_{n b}>20$. Further, the smaller the variation of the shadowing fading, the more accurate IWC can achieve for position estimation. For convenience, we use $N_{n b}=N$ in the following evaluations.

Turning to examine the effect of the number of interpolated positions $N_{\text {intp }}$, Fig. 5 presents RMSE of estimation versus the number of $N_{\text {intp }}$ for IWC under various total number of anchors $N$. The shadowing fading $\sigma_{d B}$ is set to 0,4 and $8 \mathrm{~dB}$ respectively. We found that the estimation RMSE monotonically decreases indicating that the location accuracy monotonically increases as $N_{i n t p}$ increases, and the difference is trivial when $N_{\text {intp }}>400$. To reduce the effect of $N_{\text {intp }}$ on the location accuracy as much as possible, we choose $N_{\text {intp }}=400$ in the following evaluations. 


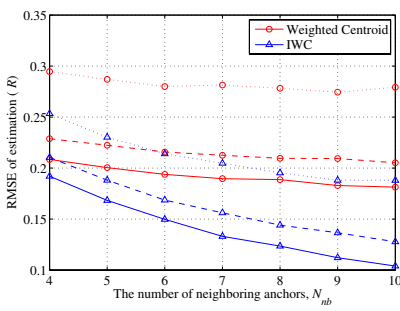

(a) $N=10$

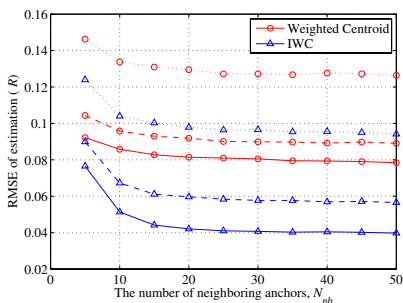

(c) $N=50$

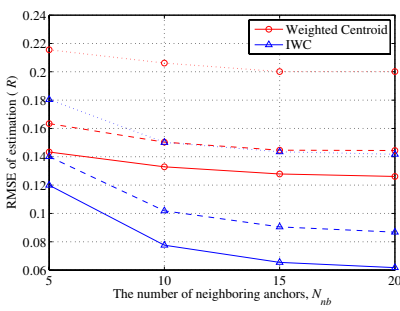

(b) $N=20$

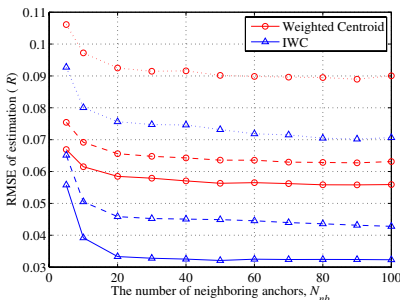

(d) $N=100$
Fig. 4. IWC: RMSE of estimation vs. the number of neighboring anchors $N_{n b}$. The total number of anchors is $N . N_{\text {intp }}$ is fixed to 500. Path loss exponent $\gamma=3.8$. The correlation distance of the shadowing $X_{C}=0 \mathrm{~m}$. The solid lines are for $\sigma_{d B}=0 \mathrm{~dB}$; the dashed lines are for $\sigma_{d B}=4 \mathrm{~dB}$; the dotted lines are for $\sigma_{d B}=8 \mathrm{~dB}$.

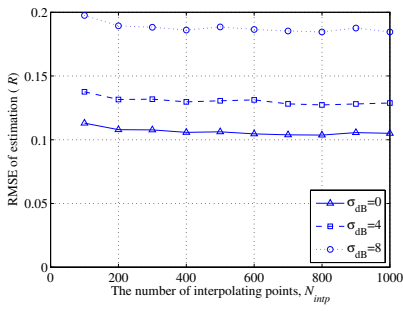

(a) $N=10$

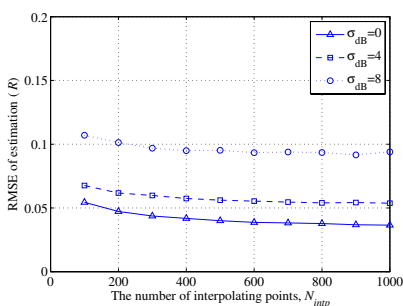

(c) $N=50$

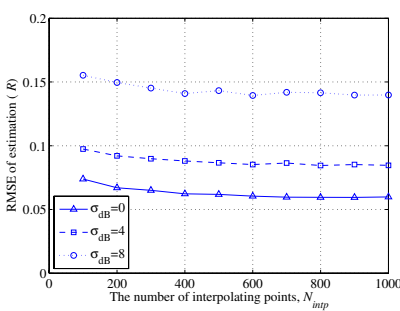

(b) $N=20$

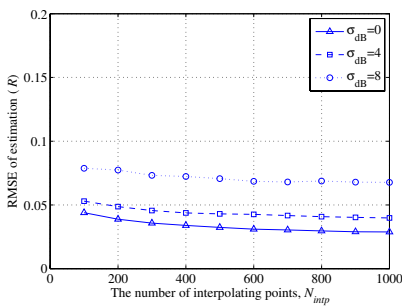

(d) $N=100$
Fig. 5. IWC: RMSE of estimation vs. the number of interpolating points

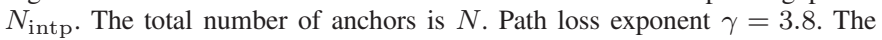
correlation distance of the shadowing $X_{C}=0 \mathrm{~m}$.

\section{Localization Performance Comparison}

We compare the performance of our proposed algorithms, IWC and IPIT, to conventional localization methods including Multilateration, Centroid and Weighted Centroid. Fig. 6 shows the estimation RMSE when varying the number of anchors in a perfect channel (i.e., $\sigma_{d B}=0 \mathrm{~dB}$ ) and under moderate shadowing conditions (i.e., $\sigma_{d B}=6 \mathrm{~dB}$ ) with no shadowing correlation $X_{C}=0$ and with shadowing correlation $\left(X_{C}=\bar{D}\right.$ and $\left.X_{C}=10 \bar{D}\right)$ respectively. $\bar{D}=\sqrt{\pi R^{2} / N}$ is the average

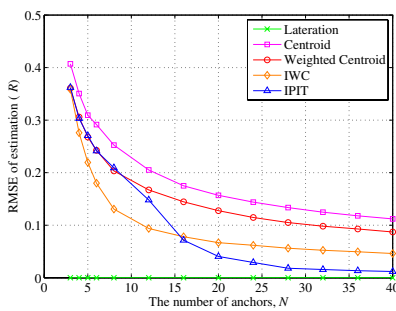

(a) $\sigma_{d B}=0 \mathrm{~dB}$

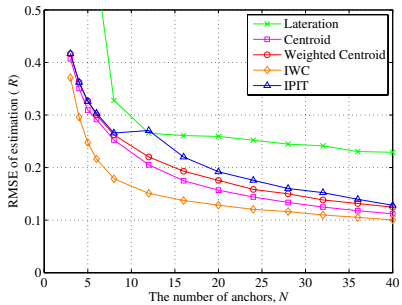

(c) $\sigma_{d B}=6 \mathrm{~dB}, X_{C}=\bar{D}$

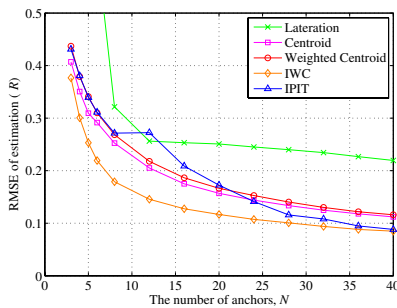

(b) $\sigma_{d B}=6 \mathrm{~dB}, X_{C}=0$

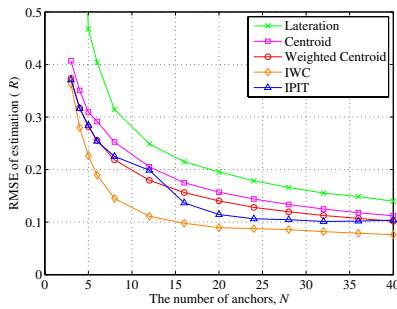

(d) $\sigma_{d B}=6 \mathrm{~dB}, X_{C}=10 \bar{D}$
Fig. 6. Comparison of RMSE across different algorithms under different number of anchors $N$. Path loss exponent $\gamma=3.8$. Average anchor spacing $\bar{D}=\sqrt{\pi R^{2} / N}$.

anchor spacing. For the Multilateration algorithm, we chose 6 anchors with the strongest RSS to construct the least square solution, as the simulation showed that Multilateration has its best performance when using about 6 to 10 anchors (results are not reported here). Although Multilateration can accurately locate the node in a perfect channel as shown in Fig. 6 (a), it requires to know the exact channel parameters (i.e. $\gamma$ and $P\left(d_{0}\right)$ in (1)). Further, the performance of localization using Multilateration decreases significantly and is worse than other algorithms under moderate shadowing conditions as shown in Fig. 6 (b), (c), and (d).

Further, we observed that when the channel is deterministic, that is, there is no random variation, the IPIT algorithm achieves the best accuracy for a sufficient number of anchors (e.g., $N \geq 20$ ) as presented in Fig. 6 (a). However, shadowing with a moderate correlation distance (e.g., $X_{C}=\bar{D}$ ) can deteriorate the accuracy of IPIT and its performance is worse than the simple centroid method at $\sigma_{d B}=6 \mathrm{~dB}$ as shown in Fig. 6 (c). Based on the results in Fig. 6 (c) and (d), we found that, in a moderate shadowing environment, IWC outperforms the other algorithms regardless of the shadowing correlation.

Fig. 7 presents the estimation RMSE of various localization algorithms as a function of $\sigma_{d B}$ with no shadowing correlation and with shadowing correlation $X_{C}=\bar{D}\left(\bar{D}=\sqrt{\pi R^{2} / N}\right)$ respectively. The simulation results demonstrate that our two interpolation based algorithms, IWC and IPIT, outperform the conventional methods for $\sigma_{d B}$ up to $8 \mathrm{~dB}$ under an uncorrelated shadowing condition as shown in Fig. 7 (a). When the shadowing is correlated by $X_{C}=\bar{D}$ as presented in Fig. 7 (b), the performance of the IWC algorithm dominates in the moderate shadowing range where $\sigma_{d B}$ is between $3 \mathrm{~dB}$ and $7 \mathrm{~dB}$, while the IPIT algorithm performs the best in a mild shadowing environment where $\sigma_{d B}$ is less than $3 \mathrm{~dB}$. 


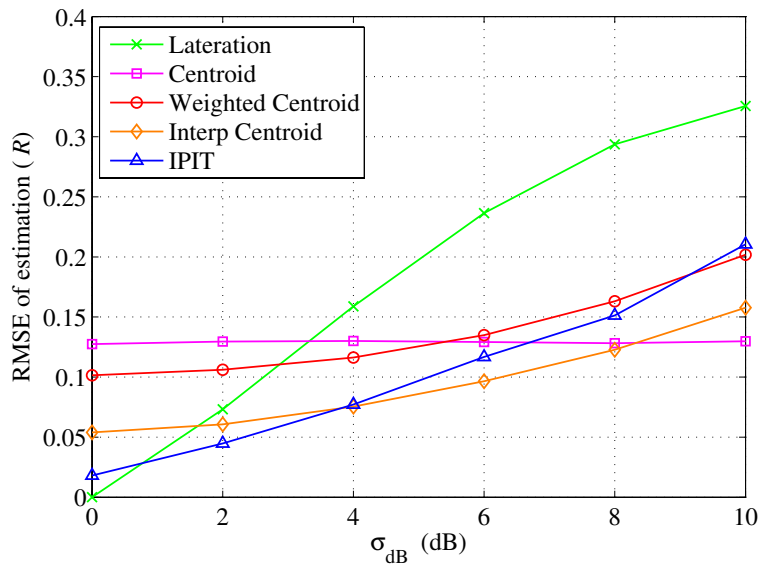

(a) $X_{C}=0$

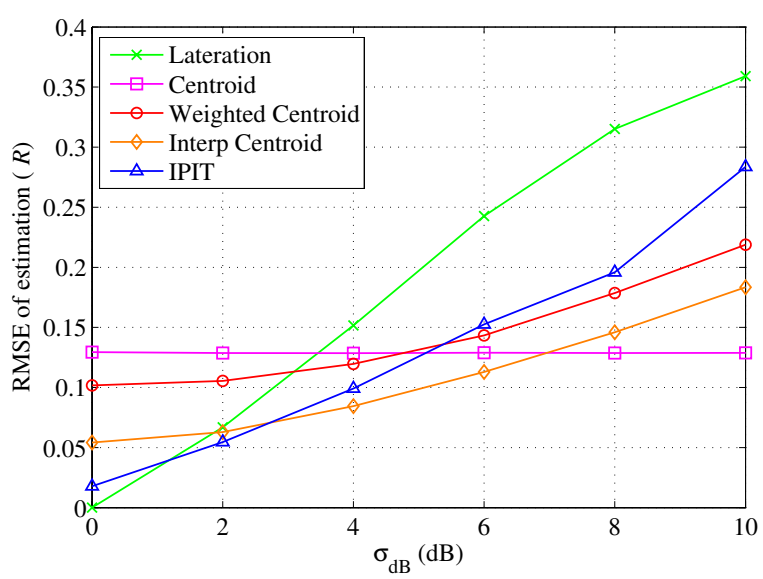

(b) $X_{C}=\bar{D}$

Fig. 7. RMSE of estimation vs. $\sigma_{d B}$. Path loss exponent $\gamma=3.8 . N=30$ Average anchor spacing

It is worth noting that, although the Multilateration method outperforms our proposed algorithms for a very small $\sigma_{d B}$, it requires to know the exact channel parameters that are usually not accessible in practice.

\section{TESTBED RESUlts}

In this section, we evaluate the effectiveness of our algorithms in an indoor environment. We evaluated our two localization algorithms in a testbed located at the Computer Science department in CoRE building at Rutgers University. The layout of the floor is shown in Fig. 8. Hallways are shaded in grey; Office and laboratories are white. The area of the floor is $200 \mathrm{ft} \times 80 \mathrm{ft}$, which contains over 50 rooms. We placed a $802.11 \mathrm{~b}$ access point at the center of the floor shown as the red triangle. We then measured RSS from the access point at multiple locations across the floor shown as small dots. The data was collected by running the iwlist scan command once a second for 60 seconds, on a Dell laptop running Linux and equipped with an Orinoco silver card. Corresponding to our proposed algorithms, the access point was used as the node to be located and every measurement location represented an anchor. Among totally 286 measured locations, we randomly

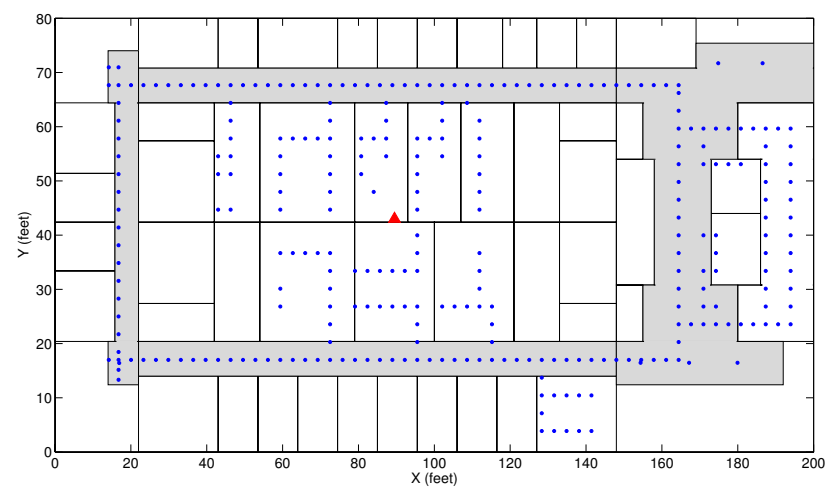

Fig. 8. The floor layout of the testbed. A $802.11 \mathrm{~b}$ access point is placed at the triangle mark as the node to be located. Received power is measured at locations shown as dots.

TABLE I

RATIO OF LOCALIZATION ERROR $(\rho(p))$ OF IWC \& IPIT TO WC IN THE TESTBED EXPERIMENT.

\begin{tabular}{|c||c|c|c|}
\hline Algorithm & 50th percentile & 70th percentile & 90th percentile \\
\hline \hline N = 30 & \multicolumn{3}{|c|}{$\rho(\mathbf{p})$} \\
\hline IWC & $64 \%$ & $63 \%$ & $64 \%$ \\
IPIT & $76 \%$ & $79 \%$ & $95 \%$ \\
\hline $\mathbf{N}=\mathbf{1 0 0}$ & \multicolumn{3}{|c|}{$\rho(\mathbf{p})$} \\
\hline IWC & $85 \%$ & $77 \%$ & $77 \%$ \\
IPIT & $71 \%$ & $70 \%$ & $73 \%$ \\
\hline
\end{tabular}

selected locations of $N=30$ and $N=100$ respectively and performed the localization test. For each value of $N$, there are more than $10^{10}$ different combinations of measurement locations. We randomly selected 2000 combinations in the test.

We compared the performance of our algorithms to a representative set of localization schemes including Nearest Neighbor, Centroid, and Weighted Centroid. In Nearest Neighbor estimation (also known as the Closest Point Approach in [20]), the node's location is approximated by the location of the anchor with the largest RSS measurement. The cumulative distribution function (CDF) of location error is shown in Fig. 9 for the 2000 measurement trials with $N$ equals to 30 and 100 respectively. For Weighted Centroid and IWC in the tests, we chose the number of neighboring anchors $N_{n b}=N$ and the number of interpolating points $N_{\text {intp }}=400$. We observed that both IWC and IPIT outperform the other algorithms for both values of $N$.

Table I presents the ratios of location error of IWC and IPIT to Weighted Centroid, respectively. The ratio of location error is defined by

$$
\rho(p)=\frac{d_{\alpha}(p)}{d_{W C}(p)},
$$

where $d_{\alpha}(p)$ is the Euclidian localization error by IWC or IPIT at the percentile $p$, and $d_{W C}(p)$ is the localization error by Weighted Centroid. Here we choose $p$ as the 50th, 70th, and 90th percentiles. We found that the values of ratio $r h o(p)$ are all less than $100 \%$ for all the percentiles under study confirming that both IWC and IPIT have better performance in 


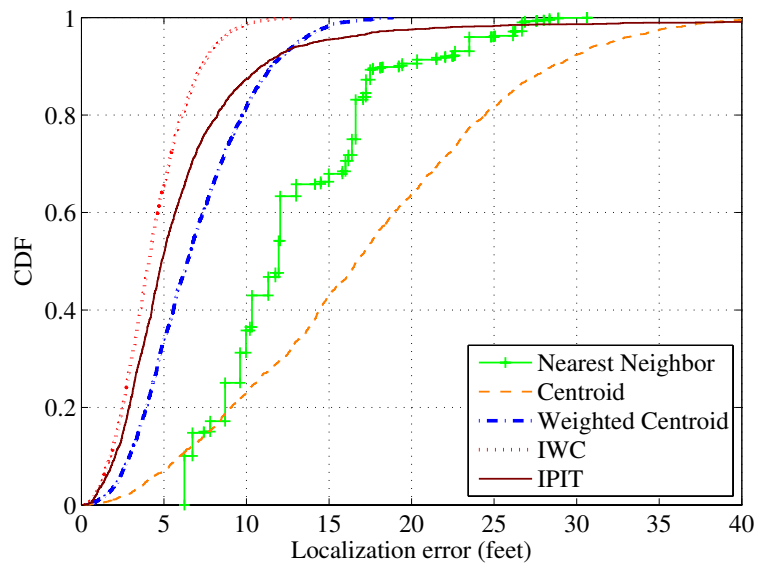

(a) $N=30$

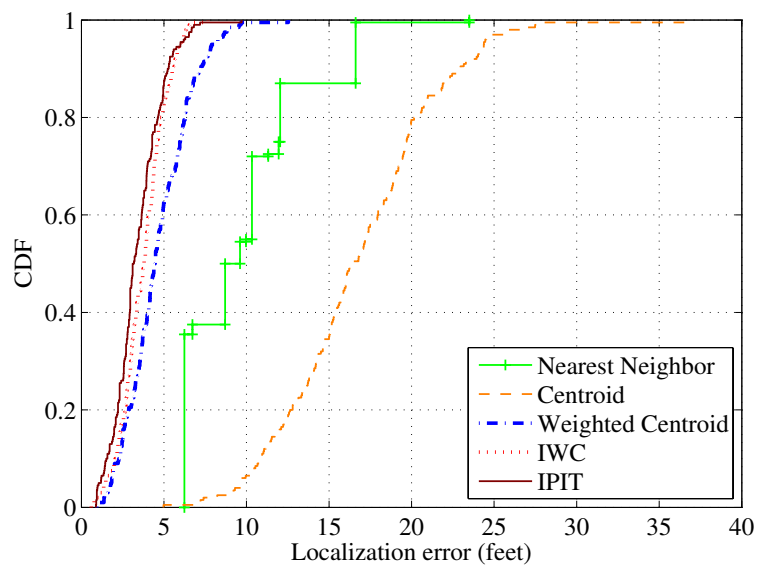

(b) $N=100$

Fig. 9. CDF of localization errors for the CoRE building testbed experiment.

localization than Weighted Centroid in indoor environments. The smaller the value of $\rho(p)$, the higher the performance improvement.

\section{CONCLUSION}

To achieve efficient spectrum sharing in the new paradigm of opportunistic spectrum access in cognitive radios, awareness of the positions of primary, secondary, or unauthorized transmitters are essential. However, cooperation between transmitters and receivers is usually not available in such a localization scenario. In this paper, we proposed two RSS based approaches, Interpolation-based Weighted Centroid (IWC) and Interpolation-based Point-In-Triangle (IPIT), which perform non-interactive localization based on dynamic signal strength mapping by using the RSS measurement in real time. The proposed methods made use of the inherent spatial correlations of the RSS measurements and recovered a fine-grained signal strength map using interpolation. Both simulation results and testbed experiments showed that the extra RSS information obtained from interpolation can help to improve the localization accuracy. Further, the effects of different number of neighboring nodes, multiple channel conditions, and various shadowing correlations have been investigated. Our results have demonstrated that our proposed approaches, IWC and IPIT, can achieve better localization accuracy than existing algorithms in both out-door as well as indoor environments.

\section{REFERENCES}

[1] W. Xu, P. Kamat, and W. Trappe, "TRIESTE: A trusted radio infrastructure for enforcing spectrum etiquettes," in Proc. Networking Technologies for Software Defined Radio Networks, 2006. SDR '06.1st IEEE Workshop on, Sep. 2006, pp. 101-109.

[2] J. K. Nelson and M. R. Gupta, "An EM technique for multiple transmitter localization," in Proc. Information Sciences and Systems, 2007. CISS '07. 41st Annual Conference on, Mar. 2007, pp. 610-615.

[3] P. Bahl and V. N. Padmanabhan, "RADAR: an in-building rf-based user location and tracking system," in Proc. INFOCOM 2000, Mar. 2000, pp. 775-784.

[4] N. Bulusu, J. Heidemann, and D. Estrin, "Gps-less low-cost outdoor localization for very small devices," IEEE Personal Commun. Mag. vol. 7, pp. 28-34, 2000

[5] T. He et al., "Range-free localization and its impact on large scale sensor networks," ACM Trans. on Embedded Computing Sys., vol. 4, pp. 877 906, Nov. 2005

[6] Y. Chen et al., "The robustness of localization algorithms to signal strength attacks: A comparative study," Distributed Computing in Sensor Systems, pp. 546-563, 2006.

[7] E. Elnahrawy, X. Li, and R. P. Martin, "The limits of localization using signal strength: a comparative study," in Proc. Sensor and Ad Hoc Communications and Networks, 2004. IEEE SECON 2004. 2004 First Annual IEEE Communications Society Conference on, Oct. 2004, pp. 406-414.

[8] M. Gudmundson, "Correlation model for shadow fading in mobile radio systems," Electronics Letters, vol. 27, pp. 2145-2146, Nov. 1991.

[9] K. Langendoen and N. Reijers, "Distributed localization in wireless sensor networks: a quantitative comparison," Computer Networks, vol. 43, pp. 499-518, 2003.

[10] L. Xiao, L. Greenstein, and N. Mandayam, "Sensor-assisted localization in cellular systems," IEEE Trans. Wireless Commun., vol. 6, pp. 4244 4248, Dec. 2007

[11] D. Niculescu and B. Nath, "Ad hoc positioning system (APS)," in Proc. Global Telecommunications Conference, 2001. GLOBECOM 'O1. IEEE, Nov. 2001, pp. 2926-2931.

[12] A. Savvides, H. Park, and M. B. Srivastava, "The bits and flops of the n-hop multilateration primitive for node localization problems," in Proc. the First International Workshop on Wireless Networks and Applications held in conjunction with Mobicom 2002, Atlanta, GA, Sep. 2002, pp. $112-121$.

[13] C. Savarese, J. M. Rabaey, and K. Langendoen, "Robust positioning algorithms for distributed ad-hoc wireless sensor networks," in Proc. the General Track: 2002 USENIX Annual Technical Conference. Monterey, CA: USENIX Association, 2002, pp. 317-327.

[14] T. He et al., "Range-free localization schemes for large scale sensor networks," in Proc. the 9th annual international conference on Mobile computing and networking. San Diego, CA: ACM, Sep. 2003, pp. 81-95.

[15] C. Liu, K. Wu, and H. Tian, "Sensor localization with ring overlapping based on comparison of received signal strength indicator," in Proc. Mobile Ad-hoc and Sensor Systems, 2004 IEEE International Conference on, Oct. 2004, pp. 516-518.

[16] Y. Shang et al., "Localization from mere connectivity," in Proc. the Fourth ACM International Symposium on Mobile Ad-Hoc Networking and Computing (MobiHoc), Jun. 2003, pp. 201-212.

[17] V. Erceg et al., "An empirically based path loss model for wireless channels in suburban environments," IEEE J. Sel. Areas Commun., vol. 17, pp. 1205-1211, Jul. 1999.

[18] V. Graziano, "Propagation correlations at $900 \mathrm{mhz}$," Vehicular Technology, IEEE Transactions on, vol. 27, pp. 182-189, 1978.

[19] H. Wendland, Scattered data approximation. New York, NY: Cambridge University Press, 2005

[20] X. Sheng and Y.-H. Hu, "Maximum likelihood multiple-source localization using acoustic energy measurements with wireless sensor networks," Signal Processing, IEEE Transactions on, vol. 53, pp. 44-53, 2005. 